\title{
Environnement et développement : la culture de la filière $\mathrm{ONU}$
}

Lucie Sauvé, Tom Berryman et Renée Brunelle

\section{(2) OpenEdition \\ Journals}

Édition électronique

URL : http://journals.openedition.org/ere/4797

DOI : $10.4000 /$ ere. 4797

ISSN : 2561-2271

Éditeur

Centr'ERE

Référence électronique

Lucie Sauvé, Tom Berryman et Renée Brunelle, « Environnement et développement : la culture de la filière ONU », Éducation relative à l'environnement [En ligne], Volume 4 | 2003, mis en ligne le 14 septembre 2003, consulté le 15 juin 2020. URL : http://journals.openedition.org/ere/4797 ; DOI https://doi.org/10.4000/ere.4797 


\title{
Environnement et développement : la culture de la filière ONU
}

\author{
Lucie Sauvé, Tom Berryman et Renée Brunelle
}

1 Après plus de 30 ans d'efforts internationaux visant à favoriser le développement de l'éducation relative à l'environnement (ERE), principalement à travers le Programme international d'éducation relative à l'environnement (PIEE) de l'UNESCO-PNUE (1975-1995), l'ERE est entrée dans une nouvelle phase d'institutionnalisation, en particulier à travers le mouvement des réformes éducatives en cours dans les différentes régions du monde. L'une des caractéristiques des nouveaux curriculums qui en émergent est en effet l'intégration d'objectifs d'apprentissage du domaine de l'éducation relative à l'environnement.

2 De façon générale, c'est surtout par la voie de la transversalité que l'environnement s'inscrit aux curriculums. C'est le cas en Belgique, en Espagne, en France et au Québec par exemple. L'environnement, présenté comme un thème intégrateur (ou "générateur ") ou encore comme un "domaine général de formation", traverse l'ensemble des matières scolaires et devient un pôle d'intégration intra-, multi- ou interdisciplinaire. Ainsi, dans le cas du Québec, l'« environnement et la consommation » constitue l'un des cinq domaines généraux de formation prescrits aux cursus du primaire et du secondaire ${ }^{1}$. Les domaines généraux de formation recouvrent divers intérêts ou besoins de l'élève et répondent à des attentes sociales jugées importantes en matière d'éducation; ils doivent être pris en compte d'une "manière synergique et interactive et constituer un tout unifié au sein d'une situation d'apprentissage » (Ministère de l'Éducation, 2001, p. 8). Dans d'autres cas, l'éducation relative à l'environnement est explicitement mentionnée comme une dimension spécifique et fondamentale d'un projet éducatif global. C'est le cas notamment en Colombie (MEN, 1994, 1998).

3 Mais souvent, dans la foulée de la plus récente proposition de l'UNESCO, celle de « l'éducation pour un avenir viable », l'environnement est présenté comme un thème parmi d'autres dans le cadre intégrateur de l'« éducation pour le développement durable ", qui devient l'une des finalités du projet éducatif. C'est le cas par exemple en Bolivie 
(República De Bolivia, 1994). Il semble que dans le contexte international actuel, caractérisé par une crise de sécurité (Jonas, 1984) et par les processus de globalisation liés à l'économicisation de notre trajectoire humaine, la reconnaissance de l'éducation relative à l'environnement (ainsi nommée ou évoquée) en tant que dimension essentielle d'un processus éducatif global, soit facilitée lorsqu'elle est associée à l'idée $\mathrm{du}$ « développement durable». Une telle association est ainsi manifeste, entre autres, dans le "domaine général de formation " «environnement et consommation » du Programme d'études québécois, où il s'agit de « construire un environnement viable dans une perspective de développement durable" (Ministère de l'Éducation, 2001, p. 46).

4 Quoi qu'il en soit, l'intégration d'une dimension environnementale dans les programmes d'études est une tâche exigeante pour les pays. À cet effet, l'inspiration et les balises des propositions internationales ont toujours été importantes, particulièrement s'il y a un besoin ou un désir d'obtenir un appui financier ou stratégique auprès des organismes internationaux, ou afin de légitimer les initiatives et d'obtenir un appui de la part d'organismes régionaux ou nationaux.

5 En raison de leur influence, il apparaît donc important d'analyser la teneur des propositions internationales au sujet de l'éducation relative à l'environnement. Quels sont leurs fondements idéologiques, épistémologiques, éthiques, curriculaires et pédagogiques? Comment sont définis les principaux concepts associés à l'éducation relative à l'environnement, en particulier «éducation", "environnement» et " développement »? Dans le but de caractériser de telles propositions et de tracer leur influence sur les réformes nationales, nous avons entrepris un programme de recherche qui s'inscrit dans le courant critique ${ }^{2}$. Ce dernier se caractérise par une démarche de déconstruction des éléments d'un ensemble, permettant d'en analyser les fondements, les composantes et les structures, afin de mieux le reconstruire (s'il y a lieu) dans une perspective de transformation et de changement social.

Dans une première phase, 29 propositions internationales liées à l'éducation relative à l'environnement ont été identifiées, émanant de diverses organisations internationales telles que l'UNESCO, le PNUE, la Banque mondiale, l'OCDE, l'UICN, etc. Ces propositions ont été systématiquement analysées (analyse de contenu lexicométrique et thématique ${ }^{3}$, afin de saisir la spécificité de chacune d'elles et d'identifier des éléments communs à l'ensemble des propositions, en tant que composantes du noyau d'une hypothétique représentation sociale globalisée de l'éducation relative à l'environnement et de ses composantes fondamentales (éducation, environnement, développement). Dans cet article, nous présenterons certains résultats de l'analyse de treize documents (tableau 1), émanant de l'Organisation des Nations Unies (ONU), sept d'entre eux provenant plus spécifiquement de l'UNESCO.

7 Les sections suivantes mettent en lumière ce que les propositions émanant de ces organismes internationaux affirment généralement ${ }^{4}$. Parallèlement, nous signalerons aussi certains aspects omis ou négligés dans les documents analysés. Ceux-ci correspondent à des propositions relevant d'autres écoles de pensée du domaine de l'éducation relative à l'environnement. Une telle identification d'aspects négligés ne vise toutefois pas l'exhaustivité: il s'agit simplement d'éclairer certaines zones d'ombre ${ }^{5}$ dans les propositions internationales afin de signaler certains pièges possibles et de repérer quelques idées orphelines du discours dominant. 
Tableau 1 : Propositions internationales liées à l'ERE étudiées dans cet article

\begin{tabular}{|c|c|c|c|}
\hline Année & $\begin{array}{l}\text { Lieu (siapplicable) } \\
\text { ef organisation }\end{array}$ & Événement (sil y a lieu) & Documents associés \\
\hline 1972 & $\begin{array}{l}\text { Stodkholm } \\
\text { ONU }\end{array}$ & $\begin{array}{l}\text { Conférence des Nations Unies sur } \\
\text { lenvironnement humain }\end{array}$ & $\begin{array}{l}\text { Délanation et plan dacction de la } \\
\text { Conference de Nations Unies sur } \\
\text { lenvironnement humain }\end{array}$ \\
\hline 1974 & $\begin{array}{l}\text { New York } \\
\text { ONU }\end{array}$ & $\begin{array}{l}\text { Assemblée gennérale - Sixième } \\
\text { session speciale }\end{array}$ & $\begin{array}{l}\text { Dedanation et programme diaction sur } \\
\text { ba mise en place dun nouvel ordre } \\
\text { ecconomigue mondial }\end{array}$ \\
\hline 1975 & $\begin{array}{l}\text { Belgrade } \\
\text { UNESCO-PNUE }\end{array}$ & $\begin{array}{l}\text { Colloque international sur } \\
\text { P'éduction relative à } \\
\text { l'environnement }\end{array}$ & $\begin{array}{l}\text { La Charte de Bel londe: Un cadre } \\
\text { mondial deducation rlative a } \\
\text { lenvironnement }\end{array}$ \\
\hline 1977 & $\begin{array}{l}\text { Thilissi } \\
\text { UNESCO-PNUE }\end{array}$ & $\begin{array}{l}\text { Conference intergouvernementale } \\
\text { sur l'́ducation relative à } \\
\text { l'environnement }\end{array}$ & 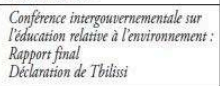 \\
\hline 1982 & $\begin{array}{l}\text { New York } \\
\text { ONU }\end{array}$ & $\begin{array}{l}\text { Assemblée générale - } 48^{\circ} \text { réunion } \\
\text { pléniere }\end{array}$ & Charte mondiale pour la nature \\
\hline 1987 & $\begin{array}{l}\text { Moscou } \\
\text { UNESCO-PNUE }\end{array}$ & $\begin{array}{l}\text { Congrès international UNESCO- } \\
\text { PNUE sur l'́duction et la } \\
\text { formation relatives ̀̀ } \\
\text { l'environnement }\end{array}$ & 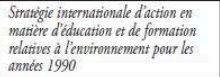 \\
\hline 1992 & $\begin{array}{l}\text { Toronto } \\
\text { UNESCO }\end{array}$ & 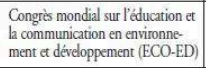 & $\begin{array}{l}\text { Refontede deleducation pour un } \\
\text { developpement durable } \\
\text { (par L.Albala-Bertrand) }\end{array}$ \\
\hline 1992 & $\begin{array}{l}\text { Rio de Janeiro } \\
\text { ONU }\end{array}$ & $\begin{array}{l}\text { Conference des Nations Unies sur } \\
\text { lénvironnement et le } \\
\text { développement }\end{array}$ & 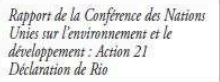 \\
\hline 1994 & $\begin{array}{l}\text { Caire } \\
\text { ONU }\end{array}$ & $\begin{array}{l}\text { Conference internationale des } \\
\text { Nations Unies sur la population et } \\
\text { le developpement }\end{array}$ & 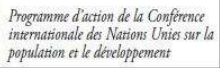 \\
\hline 1997 & $\begin{array}{l}\text { Thesslonique } \\
\text { UNESCO }\end{array}$ & 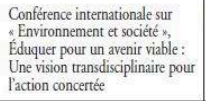 & $\begin{array}{l}\text { Rapport final } \\
\text { Declanation de Thessalonique }\end{array}$ \\
\hline 1998 & UNESCO & - & $\begin{array}{l}\text { Adaptation du contenu de leducation } \\
\text { aux deffs du XXI' siecle }\end{array}$ \\
\hline 1999 & UNESCO & - & $\begin{array}{l}\text { Lededuction et la dynamique de la } \\
\text { population: Mobbiliser les opprits pour } \\
\text { un avenir viable }\end{array}$ \\
\hline 2002 & $\begin{array}{l}\text { Johannesburg } \\
\text { ONU }\end{array}$ & $\begin{array}{l}\text { Sommet mondial pour le } \\
\text { développenent durable }\end{array}$ & $\begin{array}{l}\text { Declaration de Joharnesburg sur le } \\
\text { developpement durable } \\
\text { Plan de mise en ceurure }\end{array}$ \\
\hline
\end{tabular}

\section{L'éducation : un instrument ?}

Définir l'éducation n'est certes pas une tâche facile et cela pourrait même avoir pour effet pervers de fixer et réifier certaines idées qui doivent demeurer dynamiques et ouvertes. Mais ne pas définir l'éducation peut également mener à des résultats indésirables. À cet effet, on observe que la plupart des documents analysés ne proposent pas de définition formelle ou axiologique de l'éducation et ne discutent pas des enjeux liés aux significations diverses et parfois divergentes de l'éducation. Au lieu de cela, ils identifient une finalité pressante, celle du développement, qui permettra de résoudre les problèmes sociaux et environnementaux. L'éducation devient essentiellement un instrument de résolution de problèmes, au service du développement, plus récemment caractérisé comme développement durable. Tous les systèmes éducatifs de la planète sont invités, avec beaucoup d'insistance, à être réformés en ce sens.

9 Lorsque les documents analysés présentent certains éléments de clarification de ce qu'est l'éducation, il s'agit habituellement d'une brève proposition humaniste, qui fait référence au développement des personnes et des groupes sociaux. On observe toutefois que celle-ci n'est généralement pas cohérente avec les multiples prescriptions qui jalonnent les pages suivantes et qui indiquent une manière spécifique d'éduquer afin d'agir sur l'environnement, ou plus précisément, de résoudre les problèmes de gestion des ressources. Par exemple, si la Déclaration de Thessalonique avance une vision humaniste de l'éducation, le Rapport final de la Conférence témoigne pour sa part d'une vision instrumentale de l'éducation, au service de finalités prédéterminées. À cet effet, comparons entre elles les deux citations suivantes: 
L'éducation est un moyen indispensable pour faire en sorte que chaque femme et chaque homme dans le monde puissent maittriser son destin, exercer son choix et ses responsabilités, apprendre tout au long de la vie, sans frontières, qu'elles soient géographiques, politiques, culturelles, religieuses, linguistiques ou sexuelles. (UNESCO, 1997, p. 2)

La trame de fond de la conférence était la nouvelle vision du rôle de l'éducation et de la sensibilisation du public dans l'atteinte de la soutenabilité, tel qu'elle a émergé pendant les dernières années. L'éducation n'est plus perçue comme un objectif en soi, mais comme un moyen d'amener des changements de comportements et de styles de vie, de disséminer des connaissances et de développer des habiletés, et enfin de préparer le public à appuyer les changements vers la soutenabilité émanant d'autres secteurs de la société. (UNESCO, 1997, p. 1)

10 Le premier propos fait écho à ceux du document Action 21 qui affirmait que "l'éducation, y compris l'enseignement de type scolaire, la sensibilisation du public et la formation, doit être considérée comme un processus permettant aux êtres humains et aux sociétés de réaliser leur plein potentiel » (CNUED, 1993). Il est difficile cependant de trouver un lien de cohérence entre une telle définition humaniste et les 230 principes d'action prédéterminée émanant de ce même document. Le chapitre 36, «Promotion de l'éducation, de la sensibilisation du public et de la formation », est inclus dans la section au titre révélateur: "Moyens d'exécution". Cette section comprend également des chapitres tels que "Ressources et mécanismes financiers", "Transfert de techniques écologiquement rationnelles ", " Coopération et création de capacités ", «La science au service d'un développement durable », etc. L'éducation "revêt une importance critique pour ce qui est de promouvoir un développement durable » (CNUED, 1993); il importe donc de "réorienter l'éducation » à cet effet (CNUED, 1993).

11 Sans entrer dans une discussion sur la nature et la fonction de l'éducation, en tant que processus (impliquant le choix d'une finalité) ou en tant qu'instrument, il importe pour le moins de constater que les propositions internationales considèrent souvent l'éducation comme un instrument au service d'une finalité déterminée. Fondamentalement, cette conception instrumentale vise à former des personnes aptes à mettre en application un agenda émanant de zones de pouvoir exogènes (d'en haut, d'ailleurs), et à préparer le public à opérer des changements vers la « soutenabilité ». Dans cette perspective, un récent document de l'UNESCO porte le sous-titre évocateur « Mobiliser les esprits pour un avenir viable » (UNESCO, 1999).

Cette tendance instrumentale est d'autant plus évidente dans les propositions qui traitent de la formation des personnes, réduites - ou élevées, selon la vision adoptée au statut de "ressources humaines" (CNUED, 1993; ONU, 1995) et de "capital humain » (Albala-Bertrand, 1992 ; CNUED, 1993), coïncidant avec la montée de l'idée de développement durable suite à la conférence de Rio en 1992.

La fonction d'une éducation répondant aux besoins du développement durable consiste essentiellement à développer les ressources humaines, à encourager le progrès technique et à promouvoir les conditions culturelles favorisant les changements sociaux et économiques. Ceci est la clé de l'utilisation créatrice et effective du potentiel humain et de toutes les formes de capital pour assurer une croissance économique rapide et plus équitable tout en réduisant les incidences sur l'environnement. (Albala-Bertrand, 1992)

[...] chaque enfant devrait acquérir la connaissance, les compétences et les attitudes nécessaires à son développement personnel dans une société mondialisée, et 
devenir un membre actif d'un marché en évolution rapide. (Bureau international d'éducation, 1998, p. 11) même. Ce discours est nettement marqué par une idéologie néo-libérale et la transposition d'une logique entrepreneuriale de fourniture de services, tel que le constate également Christian Laval et Louis Weber (2000) :

[...] rentabiliser les investissements, diminuer les coûts, former à la flexibilité et à l'adaptabilité, entretenir l'employabilité. S'y ajoutent des notions comme le capital humain et le capital social. Ce qui, au total, permet de percevoir les contours et les hiérarchies d'un nouvel ordre éducatif mondial. L'OMS, la Banque mondiale, l'OCDE et la Commission européenne voient d'abord dans l'éducation et la formation un instrument au service de l'économie. [...] La mondialisation libérale de l'éducation n'est pas une catastrophe naturelle. Elle est le résultat d'une politique choisie par les gouvernements. La combattre, c'est aussi vouloir une société plus humaine et plus juste dans laquelle l'éducation est un droit fondamental, un bien public accessible à toutes et à tous.

Peut-on s'étonner que le mouvement des réformes éducatives contemporaines soit aussi marqué par cette rhétorique néo-libérale? Les diverses réformes éducatives nationales présentent en effet un certain nombre de caractéristiques communes. Bien que l'amélioration de la "qualité de l'éducation» soit présentée comme l'objectif global, on peut observer qu'une telle qualité est souvent mise en relation avec des questions économiques et politiques: on adopte une logique de rationalisation budgétaire, d'augmentation de la productivité (en termes de main d'œuvre et d'échanges économiques) et de décentralisation gouvernementale. Par ailleurs, on souhaite que l'école soit au diapason de la société actuelle, qu'elle réponde à ses besoins, en particulier, ceux du marché du travail. À cet effet, Carnoy (1999) observe que la décentralisation et la privatisation sont souvent vues comme des stratégies efficaces pour assurer la flexibilité et l'adaptabilité de l'éducation tout en améliorant sa « productivité » dans le contexte de la mondialisation des marchés, qui à son tour tend à favoriser un rôle de promotion de la croissance économique chez les États plutôt qu'un rôle de promotion de l'éducation en soi. Ce même auteur souligne d'ailleurs les caractéristiques suivantes de la globalisation dans le domaine de l'éducation.

La globalisation tend à éloigner les gouvernements des réformes basées sur l'équité pour deux raisons principales. La première est que la globalisation augmente les retombées pour les compétences « de haut niveau » versus les compétences « de bas niveau ", réduisant ainsi la complémentarité entre les réformes basées sur l'équité et celles basées sur la compétitivité. La seconde est que dans la plupart des pays en développement et dans plusieurs pays développés, les réformes de type financier dominent les changements éducatifs; dans le nouvel environnement économique mondialisé, de telles réformes tendent à augmenter le manque d'équité dans la livraison des services éducatifs. (Carnoy, 1999, p. 46, traduction libre)

Alors que les propositions internationales analysées tendent à concevoir l'éducation comme un outil pour atteindre des buts prédéfinis, ce qui demeure dans l'ombre de cette visée instrumentale est la nécessité de réfléchir de façon critique sur les réalités associées à l'environnement et au développement, et aux diagnostics généralement posés à leur sujet. Encore davantage laissée dans l'ombre est l'idée d'une éducation stimulant la réflexion sur les notions mêmes d'environnement et de développement. Avec l'affirmation constante de la nécessité d'apprendre à agir de toute urgence pour résoudre les problèmes, on éclipse l'idée que l'éducation implique la réflexivité. En fait, le mot penser n'apparaît jamais dans le chapitre XI du Programme d'action de la 
Conférence internationale des Nations Unies sur la population et le développement (ONU, 1995) traitant de l'éducation; il en va de même pour le chapitre 36 d'Action 21 (CNUED, 1993). Les mots "réflexivité " et "réfléchir ", ou l'expression "pensée critique " sont totalement absents des deux documents. Enfin, si la récente proposition de l'UNESCO (1999), L'éducation et la dynamique de la population: Mobiliser les esprits pour un avenir viable, fait appel à « un enseignement qui apprend l'art de la pensée critique, les techniques nécessaires à l'acquisition des connaissances et le devoir d'agir de façon éthique et responsable à l'égard d'autrui » (UNESCO, 1999, p.5), elle laisse place par ailleurs à une certaine forme d'endoctrinement :

L'éducation contribue de plusieurs façons à la durabilité. Elle joue un rôle particulièrement important en faisant accepter des idées nouvelles qui font appel à des concepts peu familiers et exigent des modes de pensée inhabituels. [...]. On ne peut œuvrer à la durabilité que dans la mesure où l'on persuade des millions de personnes de penser différemment et de rechercher les valeurs correspondantes. En fin de compte, la majeure partie de l'humanité doit être prête [...] à entreprendre la difficile remontée vers la durabilité. (UNESCO, 1999, p. 45)

Au bilan, la nature de la proposition éducative des documents internationaux analysés émanant de l'ONU et de l'UNESCO, en relation avec l'éducation relative à l'environnement ou avec l'éducation pour un avenir viable, est certes troublante, même si l'on reconnaît que les buts de ces propositions sont souvent davantage stratégiques qu'éducatifs ${ }^{6}$. Le tableau 2 synthétise les principaux résultats de notre analyse.

Tableau 2 : L'éducation dans les propositions internationales abordant les questions d'éducation, d'environnement et de développement

\begin{tabular}{|c|c|}
\hline \multicolumn{2}{|c|}{ ÉDUCATION } \\
\hline $\begin{array}{l}\text { Les propositions internationales mettent } \\
\text { généralement l'accent sur les aspects suivants : }\end{array}$ & $\begin{array}{l}\text { Les propositions internationales négligent } \\
\text { généralement les aspects suivants: }\end{array}$ \\
\hline 1. Léducation comme instrument. & $\begin{array}{l}\text { 1. Léducation comme processus de développement } \\
\text { humain. }\end{array}$ \\
\hline $\begin{array}{l}\text { 2. L'éducation vise l'action urgente pour résoudre des } \\
\text { problèmes. }\end{array}$ & $\begin{array}{l}\text { 2. L'éducation vise la réflexivité à l'égard des réalités } \\
\text { environnementales et du développement. }\end{array}$ \\
\hline 3. On doit adhérer à un consensus. & $\begin{array}{l}\text { 3. On doit favoriser le développement et l'exercice de } \\
\text { la pensée critique. }\end{array}$ \\
\hline $\begin{array}{l}\text { 4. L'ensemble du système éducatif doit être réformé. } \\
\text { La perspective proposée peut grandement } \\
\text { contribuer au renouvellement du système éducatif. }\end{array}$ & $\begin{array}{l}\text { 4. Il importe de tenir compte des leçons du passé et } \\
\text { ainsi reconnaitre, conserver et promouvoir des } \\
\text { expériences et initiatives éducatives pertinentes. }\end{array}$ \\
\hline
\end{tabular}

\section{L'environnement : un problème de ressources ?}

17 Si l'éducation est très peu définie dans les documents internationaux étudiés, on y trouve par contre davantage d'éléments de définition de l'environnement. Deux principales observations ressortent de notre analyse à ce sujet: d'une part, on trouve généralement une définition large de l'environnement, comme dans la Charte de Belgrade où « l'éducation relative à l'environnement devrait considérer dans sa totalité cet environnement - naturel et crée par l'homme, écologique, politique, économique, technologique, social, législatif, culturel et esthétique » (UNESCO-PNUE, 1976) ; d'autre part, malgré l'ampleur d'une telle définition initiale, l'éducation relative à 
l'environnement reste ancrée dans la conception étroite d'un environnement réduit à un ensemble de problèmes, principalement des problèmes de ressources qu'il importe de gérer plus efficacement. La Charte de Belgrade énonce ainsi que :

le but de l'éducation relative à l'environnement est de former une population mondiale consciente et préoccupée de l'environnement et des problèmes qui s'y rattachent, une population qui ait les connaissances, l'état d'esprit, les motivations et le sens de l'engagement qui lui permettent de travailler individuellement et collectivement à résoudre les problèmes actuels, et à empêcher qu'il ne s'en pose de nouveaux. (UNESCO-PNUE, 1976)

Le Rapport final de la Conférence de Tbilissi témoigne également d'une conception de l'environnement " problème » :

Pour tenter de résoudre les problèmes de l'environnement, il faut d'abord les soumettre à une analyse approfondie. Trop souvent ces problèmes ont été abordés un par un au lieu d'être envisagés dans une perspective holistique permettant d'appréhender les relations qui les unissent. La première étape du travail d'analyse devrait consister à établir une classification des types de dommages et de menaces auxquels est exposé l'environnement ou dont il peut être la cause, en tenant compte de la gravité du préjudice pouvant en résulter pour l'humanité. (UNESCOPNUE, 1977, p. 11-12)

19 Les propositions se concentrent ainsi sur des problèmes et la plupart du temps, l'environnement y est considéré comme un réservoir des ressources. On retrace un tel fil conducteur ressourciste dès la Conférence de Stockholm :

La capacité de la planète à produire des ressources renouvelables vitales doit être maintenue et, lorsque praticable, restaurée ou améliorée. [...] Les ressources non renouvelables de la planète doivent être utilisées de façon à éviter les dangers de leur déperdition future et à assurer que les bénéfices d'une telle utilisation soient partagés par toute l'humanité. (ONU, 1972, principes 3 et 5 , traduction libre)

Vingt ans plus tard, Action 21 souligne à son tour le « rôle crucial de l'environnement comme source de capital à l'état naturel et comme dépôt des résidus de la production humaine de capital et d'autres activités humaines " (CNUED, 1993). La même tendance se manifeste aussi dans d'autres documents récents de l'ONU, par exemple dans la Déclaration du millénaire des Nations Unies :

Il convient de faire preuve de prudence dans la gestion de toutes les espèces vivantes et de toutes les ressources naturelles, conformément aux préceptes du développement durable. C'est à cette condition que les richesses incommensurables que la nature nous offre pourront être préservées et léguées à nos descendants. (ONU, 2000, p. 2)

On observe aussi que dans la plupart des propositions récentes, la notion d'environnement elle-même est de moins en moins utilisée et développée. La notion de développement durable ou celle de soutenabilité tend à la remplacer : l'environnement n'y est considéré que comme l'une des dimensions en cause. La ligne d'« évolution » est manifeste : de conférences sur l'environnement dans les années 1970, on est passé à des conférences sur l'environnement et le développement dans les années 1990 puis en 2002, le Sommet mondial de Johannesburg a consacré la proposition du développement durable. Dans la Déclaration issue de ce Sommet, on constate que le mot environnement et ses dérivés (« environnementaux » par exemple) apparaît 4 fois (outre les mentions associées à des conférences antérieures) alors que l'appellation "développement durable» y apparaît 21 fois (outre les mentions associées à des conférences antérieures). Cette Déclaration affirme que «le développement économique, le développement social et la protection de l'environnement» sont des «piliers 
interdépendants et complémentaires du développement durable » (ONU, 2002, p. 1). Un peu plus loin, on constate que l'environnement est limité à un «stock de ressources naturelles nécessaires au développement économique et social", à une condition préalable au développement durable :

L'élimination de la pauvreté, l'adaptation des modes de consommation et de production, ainsi que la protection et la gestion viable du stock de ressources naturelles nécessaires au développement économique et social sont des objectifs primordiaux du développement durable et en sont aussi les conditions préalables. (ONU, 2002, p. 2)

Ainsi, dans l'ensemble des documents analysés, l'environnement est conçu comme un ensemble de problèmes et comme un réservoir de ressources; il est considéré comme soutien au développement. Or les propositions internationales ne devraient-elles pas considérer d'autres possibilités de représentations de l'environnement, telles que "l'environnement nature ", «l'environnement milieu de vie » ou "l'environnement projet communautaire à mener dans une perspective critique et politique " (Sauvé, 1996, 2002) ? L'une des premières activités en éducation relative à l'environnement ne devrait-elle pas être celle d'explorer les diverses significations et possibilités liées à la notion contemporaine d'environnement? D'où vient cette notion? Quels sont les différentes représentations de l'environnement et les divers modes de relation à ce dernier ? Qui dit quoi et qui agit comment en matière d'environnement ? Pourquoi ?

Une autre caractéristique des propositions internationales analysées est leur anthropocentrisme manifeste comme en témoigne le titre de la section d'Action 21 qui traite des questions environnementales: "Conservation et gestion des ressources aux fins du développement » (CNUED, 1993). La biosphère est destinée à nous servir, elle est vouée au développement: il s'agit d'améliorer la productivité de ses ressources, d'augmenter la productivité de la nature comme source de capital (Albala-Bertrand, 1992). D'autres manières d'interagir avec l'environnement, comme celle proposée par l'éthique de la terre d'Aldo Leopold, ou l'écologie profonde d'Arne Naess, ou l'hypothèse Gaïa de Lovelock ou encore le postmodernisme radical (grassroots postmodernism) d'Esteva et de Prakash, ne sont pas considérées. Une telle posture anthropocentrique à prétention planétaire ne devrait pas échapper à l'attention critique des éducateurs qui développent des projets liés à des contextes culturels et des milieux environnementaux particuliers, avec des individus et des groupes spécifiques, et au regard de problématiques et de réalités diverses : les nombreux enjeux reliés aux différences à l'intérieur et entre les classes sociales, les groupes d'âge, les cultures et les nations ont tendance à être évacués dans un anthropocentrisme occidental global visant l'humanité toute entière. L'anthropoéthique (certes légitime au premier degré d'une éthique d'abord centrée sur le "proche ", comme le propose J. Baird Callicott, 1989) laisse dans l'ombre d'autres propositions éthiques qui élargissent le spectre des différentes façons de construire son rapport au monde.

Par ailleurs, bien que les documents analysés reconnaissent sommairement l'importance des dimensions sociales des problématiques contemporaines, ils s'appuient fortement sur les sciences et plus précisément, sur les sciences de l'environnement et les transferts technologiques, comme des clés pour la résolution de problèmes environnementaux et pour l'éducation relative à l'environnement. Même lorsque les propositions soulignent l'importance de tenir compte de la société, de l'environnement, de l'économie et du développement, et de l'intégration de ces divers pôles entre eux, elles ne traitent pas longuement du sujet ni des méthodes pour ce 
faire. En fin de compte, les sciences sociales et la psychologie sont la plupart du temps réduites à de simples moyens de mobilisation des personnes.

Autre constat: la notion d'urgence transpire des propositions étudiées, qui adoptent toutes sensiblement la même analyse de la situation. On peut en effet observer un patron récurrent: les propositions tiennent compte du progrès accompli depuis la dernière conférence ou depuis le dernier rapport, avec l'enchaînement suivant :

1. De bons efforts ont été mis en œuvre.

2. Cependant, les résultats atteints ne sont pas suffisants.

3. La situation se dégrade.

4. Donc, il y a urgence.

On comprend ainsi l'impatience exprimée dans les propositions, à l'égard des changements environnementaux. Ceci explique également pourquoi les propositions tendent à se concentrer de plus en plus sur des actions, des résultats, des compétences et des changements de comportement, en laissant dans l'ombre la réflexivité et la pensée critique. La Déclaration de Thessalonique souligne ainsi :

que, pour réaliser l'objectif de viabilité, un immense travail de coordination et d'intégration des efforts est nécessaire dans un certain nombre de secteurs clés, de même qu'une modification rapide et radicale des comportements et des modes de vie, incluant une évolution des modes de production et de consommation. Il est indispensable à cet effet de reconnaître qu'une éducation et une sensibilisation du public appropriées constituent un des piliers de l'action en faveur de la viabilité, aux côtés de la législation, de l'économie et de la technologie. (UNESCO, 1997, p. 1-2)

En raison de ce sentiment d'urgence et de cette impatience, toutes les populations des diverses régions du monde sont appelées à s'investir rapidement et massivement dans l'agenda avancé par les propositions internationales. Il faut entre autres enrôler les enfants pour l'environnement et le développement. Cependant, l'idée inverse de fournir des environnements sains ainsi que des expériences environnementales riches et diversifiées pour le développement des enfants est généralement absente.

Enfin, nous verrons au point suivant que les propositions tendent à identifier la pauvreté comme problème principal et comme une importante cause de la détérioration de l'environnement ; le développement, associé à la croissance, apparaît comme solution première. Ici aussi, un fil conducteur se dessine de la Conférence de Stockholm à celle de Johannesburg. Toutes les propositions réclament une certaine forme de croissance économique pour résoudre les problèmes sociaux et environnementaux.

Le tableau 3 synthétise les principales caractéristiques de la notion d'environnement telles qu'elles ressortent des documents étudiés. 
Tableau 3 : L'environnement dans les propositions internationales abordant les questions d'éducation, d'environnement et de développement

\begin{tabular}{|c|c|}
\hline \multicolumn{2}{|c|}{ ENVIRONNEMENT } \\
\hline $\begin{array}{l}\text { Les propositions internationales mettent } \\
\text { généralement laccent sur les aspects suivants : }\end{array}$ & $\begin{array}{l}\text { Les propositions internationales négligent } \\
\text { généralement les aspects suivants: }\end{array}$ \\
\hline 1. Définition large et englobante de l'environnement. & $\begin{array}{l}\text { 1. Définition contextucllement signifiante et } \\
\text { opérationnelle de l'environnement. }\end{array}$ \\
\hline $\begin{array}{l}\text { 2. Une conception de l'environnement comme } \\
\text { problème, apprécié pour ses ressources. }\end{array}$ & $\begin{array}{l}\text { 2. Lenvironnement considéré pour sa propre valeur } \\
\text { intrinséque et apprécíc comme nature, milieu de vie } \\
\text { ou projet communautaire partagé. }\end{array}$ \\
\hline $\begin{array}{l}\text { 3. On doit agir sur l'environnement. Il est nécessaire de } \\
\text { gérer lenvironnement. }\end{array}$ & $\begin{array}{l}\text { 3. Il importe de réfléchir sur lenvironnement et sur nos } \\
\text { rapports à celui-ci. Il est nécessaire de gérer nos } \\
\text { rapports à l'environnement. }\end{array}$ \\
\hline $\begin{array}{l}\text { 4. Anthropocentrisme : la biosphère est destinéce à servir } \\
\text { les êtres humains. }\end{array}$ & $\begin{array}{l}\text { 4. Une invitation à analyser diverses positions éthiques, } \\
\text { dont l'écocentrisme et le biocentrismc. }\end{array}$ \\
\hline $\begin{array}{l}\text { 5. Économicisation de l'environnement : de la nature au } \\
\text { capital. }\end{array}$ & 5. Écologisation de l'économic : du capital à la nature. \\
\hline $\begin{array}{l}\text { 6. Appcl aux sciences de l'environnement et aux } \\
\text { transferts technologiques. }\end{array}$ & $\begin{array}{l}\text { 6. Appel à d'autres modes d'appréhension de l'environ- } \\
\text { nement : philosophique, holistique, expérientielle, } \\
\text { littéraire ou artistique. Lenvironnement n'est pas } \\
\text { réduit à un objet extericur à investiguer et à gérer. }\end{array}$ \\
\hline 7. Valorisation des sciences et des sciences naturelles. & $\begin{array}{l}\text { 7. Valorisation de l'histoire naturelle et d'autres } \\
\text { approches d'apprentissage relatives à l'environnement } \\
\text { et aux rdations que nous entretenons avec celui-ci. }\end{array}$ \\
\hline $\begin{array}{l}\text { 8. Limportance de tenir compte des relations entre } \\
\text { société, environnement, économic et développement. }\end{array}$ & $\begin{array}{l}\text { 8. Limportance de tenir compte do dimensions ćpisté- } \\
\text { mologique, philosophique, psychologique, spirituelle et } \\
\text { dautres dimensions de nos relations à lenvironnement. }\end{array}$ \\
\hline 9. Jusquà maintenant, les résultats ne sont pas satisfaisants & 9. Certains résultats sont prometteurs. \\
\hline 10. Il y a urgence. & $\begin{array}{l}\text { 10. Il est nécessaire d'agir de façon énergique mais avec } \\
\text { prudence, rigueur et ćrconspection. }\end{array}$ \\
\hline 11. Enrốler les enfants pour l'environnement. & $\begin{array}{l}\text { 11. Fournir des environnements propices au développement } \\
\text { des enfants. Laisser les enfants s'engager dans le monde. }\end{array}$ \\
\hline $\begin{array}{l}\text { 12. La pauvreté est le problème principal et la cause } \\
\text { principale des autres problèmes. }\end{array}$ & $\begin{array}{l}\text { 12. La pauvreté est un symptôme de formes plus profondes } \\
\text { daliénation. La pauvecté contemporaine est causée par } \\
\text { lappropriation des ressources économiques et des abus } \\
\text { environnementaux et sociaux chez la minoritét } \\
\text { privilegiéc. }\end{array}$ \\
\hline $\begin{array}{l}\text { 13. Le développement et la croissance vont résoudre les } \\
\text { problèmes environnementaux. }\end{array}$ & $\begin{array}{l}\text { 13. Le développement et la croissance sont de nature à } \\
\text { contribuer aux problèmes environnementaux et sociaux. }\end{array}$ \\
\hline
\end{tabular}

\section{Le développement : une croissance économique soutenue?}

Comme le concept d'éducation, celui de développement est généralement mal défini dans les documents internationaux analysés. On en trouve rarement une définition formelle. On observe par ailleurs que la notion de développement donne lieu à une certaine incohérence semblable à celle observée à propos des notions d'éducation et d'environnement. En effet, de la même manière que la vision humaniste initiale de l'éducation se rétrécit rapidement dans l'étau d'une proposition instrumentale et que la vision englobante de l'environnement, telle qu'annoncée en début des documents, cède à une conception essentiellement ressourciste, la notion de développement, d'abord justifiée comme processus d'amélioration de la qualité de vie humaine, tend à se restreindre à la seule croissance économique. Il existe en effet une tension entre les significations accordées au développement dans les propositions analysées : d'une part, un développement humain global et d'autre part, le développement des ressources, le développement économique et la croissance économique. Ces visions divergentes du développement (qui s'avèrent en réalité conflictuelles) sont par ailleurs mal définies, ce qui ouvre la voie à une pluralité d'interprétations, parfois contradictoires et dont le caractère flou entretient la confusion. Qu'entend-on par qualité de vie? Qu'entend-on par développement humain? Dans une telle confusion, l'appréciation du développement en fonction des indicateurs quantitatifs de la croissance économique (« dollarisation ») semble exercer une forte attirance. 
31 Il importe de rappeler ici que l'éducation relative à l'environnement est née dans le creuset du « Nouvel ordre économique mondial " promulgué en 1974. La Déclaration relative à ce nouvel ordre est issue d'une session spéciale de l'Assemblée générale des Nations Unies «ayant pour but d'étudier pour la première fois les problèmes de matières premières et de développement, dans la perspective d'envisager la résolution des problèmes économiques les plus importants se posant à la communauté mondiale » (ONU, 1974, traduction libre). Ce document témoigne d'une conception de la biosphère comme réservoir de ressources pour servir la croissance économique ; l'environnement est associé à des "problèmes des matières premières" (raw material); il est ainsi étroitement relié au développement; l'environnement et le développement sont conjointement imbriqués dans les "problèmes économiques les plus importants ». La Déclaration affirme qu'un nouvel ordre économique mondial doit «corriger les inégalités et les injustices, éliminer l'écart grandissant entre les pays développés et en développement, assurer une accélération constante du développement économique et social et assurer la paix et la justice pour les générations présentes et futures » (ONU, 1974, traduction libre). La préoccupation de partage et d'équité dont témoigne ce nouvel ordre économique est certes d'importance capitale; le rapport à l'environnement qui y est proposé n'en reste pas moins étroitement utilitariste: l'environnement doit être préservé pour la pérennité de ses matières premières, afin de pouvoir poursuivre un développement continu et équitable dans le monde entier. La Charte de Belgrade s'inscrit dans un tel cadre de référence : si elle réclame de nouveaux modèles de développement, elle présente le développement comme une aventure essentiellement économique :

La récente déclaration des Nations Unies en faveur d'un Nouvel ordre économique international appelle une conception nouvelle du développement, une conception qui prenne en considération la satisfaction des besoins et des aspirations de tous les citoyens du globe, le pluralisme des sociétés, l'équilibre et l'harmonie entre l'humanité et le développement [...] les ressources du monde devraient être mises en valeur de manière à profiter à toute l'humanité et à fournir le potentiel qui permettra d'améliorer la qualité de vie de chacun. (UNESCO-PNUE, 1976)

Outre l'appel à un développement économique soucieux de la gestion des stocks de ressources, l'une des constantes du discours des organismes internationaux en matière de développement et d'environnement est de considérer la pauvreté comme le plus grave problème, en amont de tous les autres, ralentissant la croissance économique et nuisant à l'environnement.

Simultanément, le développement apparaît comme une nécessité pressante. La pauvreté est elle-même une forme de dégradation de l'environnement. Dans cette perspective, il n'est plus possible de mettre en balance la préservation de l'environnement et la nécessité du développement. Dans un grand nombre de pays les moins avancés et en particulier si l'on considère les groupes les plus désavantagés qui sont la grande majorité, le développement est un préalable à la préservation de l'environnement, notamment parce qu'il faut tâcher de satisfaire les besoins des populations les plus démunies. Dans ces conditions, les stratégies pour la préservation et l'amélioration de l'environnement coïncident, en fait, dans une large mesure, avec les efforts de développement. (UNESCO-PNUE, 1977, p. 11) La pauvreté rend difficile l'accès à l'éducation et aux autres services sociaux et favorise l'accroissement de la population et la dégradation de l'environnement. Réduire la pauvreté est donc un objectif essentiel et une condition sine qua non de la viabilité. (UNESCO, 1997, p. 2) 
33

Il semble donc que malgré la reconnaissance des problèmes liés au développement (et les propositions analysées reconnaissent l'existence de tels problèmes), la croissance économique devient un élément-clé de la solution. Entre autres, dans le Programme d'action de la Conférence internationale des Nations Unies sur la population et le développement du Caire (1995), des appels à la «croissance économique soutenue dans le cadre du développement durable» ou à la "croissance économique et au développement durable » apparaissent plus de vingt fois. Le premier élément du préambule synthétise les thèmes centraux qui sont développés dans l'ensemble du document :

Avec la reconnaissance croissante de l'interdépendance de la population, du développement et de l'environnement à l'échelle mondiale, le moment n'a jamais été aussi propice à l'adoption de politiques macro-économiques et socioéconomiques de nature à assurer une croissance économique soutenue dans le cadre du développement durable de tous les pays et à la mobilisation des ressources humaines et financières en vue de résoudre les problèmes de la planète. Jamais auparavant la communauté mondiale n'avait eu à sa disposition autant de moyens, de connaissances, de technologies aussi puissantes qui, s'ils sont convenablement réorientés, pourraient favoriser une croissance économique soutenue et un développement durable. Néanmoins, l'utilisation efficace des ressources, des connaissances et des technologies est entravée par des obstacles politiques et économiques à l'échelon national et international. En conséquence, bien que de vastes ressources soient disponibles depuis un certain temps, leur utilisation en faveur d'un développement socialement équitable et écologiquement rationnel a été très sensiblement limitée. (ONU, 1995, p. 8)

Le développement est ainsi présenté comme un droit et une obligation, fortement associé à la croissance économique soutenue. Une telle proposition exclut l'idée de considérer le développement comme une option, un choix et, à tout le moins, celle d'explorer la notion de développement et ses différentes significations et possibilités. Craindrait-on ici qu'une conception du développement comme un projet endogène et alternatif devienne l'un des « obstacles politiques et économiques » à la mise en œuvre de la solution d'une croissance soutenue globale, comme solution universelle?

Nous réaffirmons notre engagement à lutter contre les conditions universelles qui nuisent gravement au développement durable de nos peuples : la faim chronique, la malnutrition, l'occupation des territoires étrangers, les conflits armés, les problèmes de drogues illicites, et les désastres naturels. (ONU, 2002, p. 3)

On observe que parmi les problèmes mentionnés dans ce paragraphe de la Déclaration de Johannesburg, on omet de signaler les impacts négatifs du développement néolibéral, de la globalisation des marchés, des ajustements structuraux, etc. On réduit les problèmes environnementaux à des "désastres naturels». S'appuyant sur un tel diagnostic myope, la croissance économique apparaît aisément comme la solution clé. Le raisonnement peut trop facilement devenir le suivant: la croissance économique (continue et illimitée) est nécessaire pour éliminer la pauvreté, soutenir le développement humain et résoudre par le fait même les problèmes environnementaux. Un tel raisonnement mène vers l'idée que le développement humain est basé sur la croissance économique. Et puisque dans ce discours, les enjeux d'environnement et de développement sont indissociables, une nouvelle forme de croissance économique résoudra également les problèmes environnementaux de ressources. On peut ainsi lire dans la Déclaration de Johannesburg:

Nous travaillerons ensemble pour nous entraider à accéder aux ressources financières, aux avantages de l'ouverture des marchés, à développer nos capacités, à utiliser la technologie moderne pour réaliser le développement et s'assurer qu'il y 
ait transfert technologique, développement des ressources humaines, éducation et formation pour bannir à jamais le sous-développement. (ONU, 2002, p. 3) des marchés, s'impose donc dans la proposition onusienne. Or dans un contexte éducatif, l'exploration critique de la notion contemporaine et polysémique de développement (comme celle d'environnement) ne devient-elle pas une stratégie de formation de premier ordre? D'où vient cette notion du développement? Quelles sont ses différentes acceptions et significations pour différents acteurs et groupes sociaux? Comment expliquer cette diversité de conceptions? De telles questions sont pourtant éludées dans un contexte où l'éducation est vue comme un instrument pour transmettre une certaine vision du développement, celui-là même qui, en fin de compte, est évalué en fonction d'indicateurs quantitatifs et économiques.

La relation entre l'éducation et le développement (d'ordinaire défini en termes de croissance du revenu national ou du revenu par habitant) a fait l'objet de nombreuses études ces 30 dernières années et est universellement acceptée, de même que le rôle de l'éducation comme instrument d'évolution sociale et démographique. En effet, quand des économistes des années 60 se sont aperçus que l'éducation représentait un investissement pour le développement et non pas, comme on le pensait avant, une ponction sur l'économie, cette «découverte » a eu un effet puissant sur la politique d'éducation comme sur la politique de développement. De ce fait, les particuliers comme les gouvernements se sont aussi montrés plus généreux pour financer l'éducation en même temps que plus exigeants dans leurs évaluations : ils veulent connaitre la nature exacte de ce qu'ils « achètent ", son contenu, sa qualité, voire son rendement. (UNESCO, 1999, p. 47)

Ainsi la croissance économique mondiale devient maintenant la solution universelle aux problèmes contemporains et l'éducation doit être mise au service d'une telle croissance. La nouvelle équation se lit ainsi : éducation $=$ développement $=$ croissance économique $=$ solution à tous les problèmes. Le travail des éducateurs doit être rentable!

On observe enfin que toutes les propositions internationales analysées adoptent une vision de la Terre comme si elle était vue sous un télescope lunaire. Les auteurs insistent sur l'importance de la coopération, de la solidarité et de l'interdépendance. Dans l'ombre de cet appel pour une fraternité entre les délégués des nations visant un but commun, restent toutefois en retrait les notions d'autonomie et de gestion endogène. Ces dernières devraient essentiellement être liées à des projets de développement alternatifs, de type local ou biorégional, ouvrant la voie à une meilleure harmonisation des relations société-environnement. Or en matière de solidarité ou d'écodéveloppement endogène (deux propositions qui devraient être étroitement reliées), on peut douter que la proposition du « développement durable » soit adéquate quand on constate l'affirmation suivante du President's Council on Sustainable Development (1997, p. 98) des États-Unis, selon laquelle « le développement durable est un excellent moyen d'augmenter la compétitivité nationale des États-Unis dans le contexte de la globalisation actuelle ».

Le tableau 4 résume les principales caractéristiques de la notion de développement telle qu'elle se dégage des propositions internationales analysées. 
Tableau 4 : Le développement dans les propositions internationales abordant les questions d'éducation, d'environnement et de développement

\begin{tabular}{|c|c|}
\hline \multicolumn{2}{|c|}{ DÉVELOPPEMENT } \\
\hline $\begin{array}{l}\text { Les propositions internationales mettent } \\
\text { généralement l'accent sur les aspects suivants : }\end{array}$ & $\begin{array}{l}\text { Les propositions internationales négligent } \\
\text { généralement les aspects suivants : }\end{array}$ \\
\hline $\begin{array}{l}\text { 1. Le développement comme une exigence, une } \\
\text { obligation, un destin. }\end{array}$ & $\begin{array}{l}\text { 1. Le développement comme une option, un choix. } \\
\text { L'exigence est plutôt de réfléchir sur la notion de } \\
\text { développement et de justifier une conception } \\
\text { particulière. }\end{array}$ \\
\hline $\begin{array}{l}\text { 2. Le développement comme croissance économique } \\
\text { soutenue et illimitée. }\end{array}$ & $\begin{array}{l}\text { 2. Le développement comme déploiement des } \\
\text { possibilités humaines. }\end{array}$ \\
\hline $\begin{array}{l}\text { 3. Le développement comme un projet global sous une } \\
\text { gestion mondiale. }\end{array}$ & 3. Le développement comme un projet endogène. \\
\hline 4. Le développement comme la solution. & 4. Le développement comme une problématique. \\
\hline $\begin{array}{l}\text { 5. Le développement est indissociable de } \\
\text { l'environnement. }\end{array}$ & $\begin{array}{l}\text { 5. Le rapport à l'environnement peut être appréhendé } \\
\text { de diverses façons, et non nécessairement en lien } \\
\text { avec des questions de développement. }\end{array}$ \\
\hline $\begin{array}{l}\text { 6. L'économie est une force autonome à l'extérieur de } \\
\text { la société, qui détermine la relation entre la société et } \\
\text { l'environnement. }\end{array}$ & $\begin{array}{l}\text { 6. L'économie fait partie de la sphère sociale ; elle est } \\
\text { un aspect de la réalité sociale, un ensemble de choix } \\
\text { responsables. }\end{array}$ \\
\hline $\begin{array}{l}\text { 7. Limportance de la coopération, de la solidarité et de } \\
\text { linterdépendance. }\end{array}$ & $\begin{array}{l}\text { 7. Limportance de l'autonomie et de la gestion } \\
\text { endogène. }\end{array}$ \\
\hline
\end{tabular}

\section{Conclusion} l'intégration de l'éducation relative à l'environnement aux nouveaux curriculums. À cet effet, les propositions émanant d'instances internationales peuvent offrir des balises et des sources d'inspiration pour aborder les enjeux interreliés de l'éducation, de l'environnement et du développement. Jusqu'ici, l'impulsion fournie par les conférences et les documents de l'UNESCO-PNUE pour légitimer, institutionnaliser et développer l'éducation relative à l'environnement a été remarquable et a permis des avancées considérables. Les propositions qui en émanent agissent encore en tant que repères et soutien pour concevoir et mettre en œuvre des programmes et des projets dans les différentes régions du monde.

Il importe de considérer toutefois que les documents relatifs à l'éducation (dont l'éducation relative à l'environnement) produits dans le cadre des organisations internationales proposent aux nations des buts, objectifs et principes d'ordre stratégiques; or ces derniers ne devraient pas être confondus avec des finalités éducatives ou des principes pédagogiques; il ne s'agit pas non plus de credos. Ces documents ont généralement été conçus et rédigés par un auteur anonyme ou une équipe d'auteurs ; or ces personnes peuvent être compétentes et impliquées en ce qui a trait à l'environnement ou au développement, mais s'agit-il pour autant de spécialistes en éducation? Sont-elles bien documentées et possèdent-elles une culture et une expérience dans le domaine éducatif? Quel agenda adoptent-elles? Quelle est la couleur idéologique de cet agenda ${ }^{7}$ ? On observe que la perspective éducative des documents analysés est faible, particulièrement en ce qui a trait aux fondements éthiques, épistémologiques et pédagogiques des propositions. Notamment, les quelques pages écrites au sujet de l'éducation dans le Rapport Brundtland (Commission mondiale sur l'environnement et le développement, 1988), tout comme celles du chapitre 36 d' 
Action 21, correspondent à des discours éducatifs peu éclairés. Est-il étonnant alors que les acteurs de l'éducation n'aient pas été inspirés ou interpellés par ces documents?

Les propositions internationales correspondent à des balises conçues par des délégués de nations, pour guider et légitimer les choix éducationnels dans les divers pays. Prendre ces propositions à la lettre pour former tous les enfants, tous les jeunes et adultes des diverses régions du monde, dans les domaines de l'environnement et du développement est à tout le moins inquiétant. Les éducateurs et éducatrices ne doivent-ils pas adopter une perspective critique à l'égard des propositions officielles ? Que peuvent-elles offrir comme support à l'institutionnalisation et au développement de l'éducation relative à l'environnement? Quelles en sont les limites?

Cet article a tenté de saisir le fil conducteur qui relie les propositions issues des grandes conférences internationales de Stockholm, Belgrade, Tbilissi, Moscou, Rio de Janeiro, Le Caire, Thessalonique et Johannesburg 8 . Un des éléments de ce fil conducteur est un appel à l'éducation comme instrument pour mettre en application un programme d'action visant à réorienter le monde en fonction du développement durable. Les propositions internationales analysées adoptent également une vision de l'environnement qui se restreint à un ensemble de problèmes de ressources et une vision du développement étroitement associée la croissance économique, celle-ci étant une condition centrale du développement humain. L'éducation relative à l'environnement y devient un outil au service du développement durable. Or on oublie comment une telle construction de l'environnement et du développement, pourtant imposée à l'ensemble des peuples, est spécifique à la culture nord-occidentale dominante. On risque de perdre de vue qu'il est possible, voire nécessaire de questionner une telle construction, qui s'impose comme vision du monde et oriente notre rapport à ce dernier.

L'affirmation que "c'est le savoir, plus que le travail, les matières premières ou le capital, qui est devenu la ressource clé des économies modernes » (UNESCO, 1999, p. 48) requiert également une analyse critique. On peut très sérieusement douter que tout considérer comme une ressource économique offre une réponse appropriée à la résolution des problèmes socio-environnementaux contemporains, bien au contraire. Neil Evernden (1985) souligne à cet effet qu'en « décrivant quelque chose comme une ressource, cela nous donne un permis pour l'exploiter ». Dans un tel contexte, l'appel à élargir la vision, soi-disant limitée, de l'éducation relative à l'environnement, en transformant tout en ressources dans la perspective d'un développement durable (les gens = «main d'œuvre »; la nature = « matières premières »; l'argent $=$ « capital »; la connaissance $=$ "ressource-clé $»)$ ne nous propose-t-il pas une réduction fallacieuse, pourtant présentée comme une expansion libératrice ? S'il faut réduire la terre entière (les gens, les plantes, les animaux, le vent, etc.) au seul concept de ressource, cela n'appauvrit-il pas fondamentalement tout un chacun de nous, êtres vivants et nonvivants de cette "maison de vie» partagée qu'est l'environnement? Cela ne nous prive-t-il pas des multiples et diverses dimensions et significations possibles du rapport au monde? Ne s'agit-il pas d'une bien triste dérive, causée par une grave myopie idéologique, éthique et culturelle? 


\section{BIBLIOGRAPHIE}

Albala-Bertrand, L. (1992). Refonte de l'éducation : pour un développement durable. Dossier Environnement et Développement de l'UNESCO. Paris : UNESCO.

Bardin, L. (1996). L'analyse de contenu. Paris : Presses Universitaires de France. Bateson, G. (1984). La nature et la pensée. Paris : Seuil.

Bureau international d'éducation (BIE). (1998). Adaptation du contenu de l'éducation aux défis du XXIe siècle. Genève : BIE, UNESCO.

Callicott, J.B. (1989). In defense of the land ethic : Essays in environmental philosophy. New York : State University of New York Press.

Carnoy, M. (1999). Globalization and educational reform : what planners need to know. Paris : UNESCO, International Institute for Educational Planning.

CNUED. (1993). Action 21 : Rapport de la Conférence des Nations Unies sur l'environnement et le développement. Déclaration de Rio sur l'environnement et le développement. Conférence des Nations Unies sur l'environnement et le développement, Rio de Janeiro, 3-14 juin 1992. Rio de Janeiro : Commission des Nations Unies pour l'environnement et le développement (CNUED).

Commission mondiale sur l'environnement et le développement. (1988). Notre avenir à tous (Rapport Brundtland). Montréal : Éditions du Fleuve.

Delors, J. (éd.), Commission internationale sur l'éducation pour le vingt et unième siècle. (1996). L'éducation, un trésor est caché dedans : Rapport à l'UNESCO de la Commission internationale sur l'éducation pour le vingt et unième siècle. Paris : UNESCO.

Evernden, N. (1985). The natural alien : Humankind and environment. Toronto : University of Toronto Press.

Faure, E., Commission internationale sur le développement de l'éducation (1974). Apprendre à être. Paris : UNESCO.

Jonas, H. (1984). The imperative of responsibility: The search of an ethics for the technological age. Chicago : The University of Chicago Press.

Laval, C. et Weber, L. (2000). Le nouvel ordre éducatif mondial : OMC, Banque mondiale, OCDE, Commission européenne. Paris : Éditions Nouveaux Regards / Syllepse.

MEN. (1998). Lineamientos generales para una política nacional de educación ambiental. Documento de apoyo. Santafé de Bogotá : Ministerio de Educación Nacional (MEN).

MEN. (1994). Ley general de educación : Ley 115 de 1994. Santafé de Bogotá : Ministerio de Educación Nacional (MEN).

Ministère de l'Éducation. (2001). Programme de formation de l'école québécoise. Version approuvée. Éducation préscolaire. Enseignement primaire. Québec : Ministère de l'Éducation.

ONU. (2002). Déclaration de Johannesburg sur le développement durable. Sommet mondial pour le développement durable, Johannesburg, 26 août-4 septembre 2002. Johannesburg : Nations Unies. ONU. (2000). Déclaration du millénaire. Résolution 55/2 adoptée par l'Assemblée générale des Nations Unies, 55e session, 8e réunion plénière. New York : Nations Unies. 
ONU. (1995). Programme d'action de la Conférence internationale des Nations Unies sur la population et le développement. Conférence internationale des Nations Unies sur la population et le développement, Caire, 5-13 septembre 1994. New York : Nations Unies.

ONU. (1982). La charte mondiale pour la nature. Madrid : ONU.

ONU. (1974). Déclaration et programme d'action sur la mise en place d'un nouvel ordre économique mondial. Résolutions adoptées quant au Rapport du comité Ad Hoc de la 6e session spéciale, Assemblée générale, 1er mai 1974. New York : Nations Unies.

ONU. (1972). Déclaration et plan d'action de la Conférence des Nations Unies sur l'environnement humain. Conférence des Nations Unies sur l'environnement humain, Stockholm, 5-16 juin 1972. New York : Nations Unies.

Orellana, I. et Fauteux, S. (2000). L'éducation relative à l'environnement à travers les grands moments de son histoire. In A. Jarnet, B. Jickling, L. Sauvé, A. Wals et P. Clarkin (dir.), Proceedings from an On-Line Colloquium. The Future of Environmental Education in a Postmodern World? Whitehorse : Yukon College, p. 13-24.

President's Council on Sustainable Development. (1997). From classroom to community and beyond : Educating for a sustainable future. Washington, DC : PCSD.

República de Bolivia. (1994). Reforma educativa : Ley no. 1565, 7 de Julio de 1994. Bolivia : República de Bolivia.

Sauvé, L. (2002). L'éducation relative à l'environnement : possibilités et contraintes. Connexion, La revue d'éducation scientifique, technologique et environnementale de l'UNESCO, 27(1/2), 1-4.

Sauvé, L. (1996). Environmental education and sustainable development : A further appraisal. Canadian Journal of Environmental Education, 1, 7-34.

UNESCO. (1999). L'éducation et la dynamique de la population: Mobiliser les esprits pour un avenir viable. Paris : UNESCO.

UNESCO. (1997). Éduquer pour un avenir viable: Une vision transdisciplinaire pour l'action concertée. Rapport final et Déclaration. Conférence internationale de Thessalonique (Grèce), 8-12 décembre 1997. Paris : UNESCO.

UNESCO-PNUE. (1987). Stratégie internationale d'action en matière d'éducation et de formation relatives à l'environnement pour les années 1990. Congrès international UNESCO-PNUE sur l'éducation et la formation relatives à l'environnement, Moscou, 17-21 août 1987. Paris : UNESCO.

UNESCO-PNUE. (1977). Déclaration de Tbilissi et Rapport final. Conférence intergouvernementale sur l'éducation relative à l'environnement, Tbilissi, 14-26 octobre 1977. Paris : UNESCO.

UNESCO-PNUE. (1976). La Charte de Belgrade. Colloque international sur l'éducation relative à l'environnement, Belgrade, 13-22 octobre 1975. Connexion, Bulletin de l'éducation relative à l'environnement, 1, 1-3.

\section{NOTES}

1. Au Québec, les cinq domaines généraux de formation sont les suivants : Santé et bien-être, Orientation et entrepreneuriat, Environnement et consommation, Médias, Vivre ensemble et citoyenneté. 
2. Cet article présente et discute certains résultats de la recherche L'éducation relative à l'environnement et au développement dans le cadre des réformes éducatives en cours - Le cas du Québec, financée par le Conseil de recherche en sciences humaines (CRSH) du Canada.

3. Une «lecture flottante» (Bardin, 1996) a d'abord permis d'explorer globalement les propositions en question. Puis, les mentions ou références à l'éducation, à l'environnement, au développement, à l'éducation relative à l'environnement et à l'éducation pour le développement durable ont été systématiquement identifiées et analysées à l'aide de grilles spécifiques. Ont également été analysées d'autres caractéristiques des propositions: le problème principal diagnostiqué, les champs de savoirs privilégiés, les valeurs implicites et explicites privilégiées, le type de discours, la présence ou l'appel à la réflexivité et la pensée critique, le type de références, etc.

4. Un angle d'analyse complémentaire nous permettrait de situer ces affirmations dans le contexte géopolitique et socio-environnemental global des différentes périodes au cours desquelles ces propositions ont été développées. À cet effet, l'article d'Isabel Orellana et Stéphane Fauteux (2000) est éclairant.

5. Bateson (1984, p. 53) observe que « Zéro, l'absence d'élément peut contenir un message ».

6. Il faut signaler que l'UNESCO a publié par ailleurs des textes fort inspirants sur l'éducation en général, en particulier ceux d'Edgar Faure, Commission internationale sur le développement de l'éducation (1974) et de Jacques Delors (éd.), Commission internationale sur l'éducation pour le vingt et unième siècle (1996).

7. Il importe de reconnaître que les propositions émergeant des travaux des instances internationales sont aussi le résultat de tensions politiques internes et externes.

8. On observe des constantes dans les propositions internationales analysées. Les visions de l'éducation, de l'environnement et du développement sont relativement les mêmes et elles sont restées relativement stables au cours des 30 dernières années. Il y a bien sûr certaines variations et nuances. En particulier, certaines variations peuvent être observées entre les documents des Nations Unies et ceux de l'UNESCO. Cette dernière semble avoir une vision de l'éducation moins instrumentale, une vision de l'environnement moins réduite à des problèmes de ressources et une vision du développement moins restreinte à la croissance économique. Il serait intéressant d'étudier ces nuances et différences au fil des prochaines années, alors que l'UNESCO s'est vue confier le mandat de l'application du chapitre 36 d'Action 21.

\section{RÉSUMÉS}

L'impulsion fournie par les conférences et les documents de l'UNESCO-PNUE pour légitimer, institutionnaliser et développer l'éducation relative à l'environnement (ERE) est certes remarquable. Les propositions qui en émanent agissent encore en tant que balises pour concevoir et mettre en œuvre des programmes et des projets dans les différentes régions du monde. En raison de l'importance d'un tel appui et d'une telle influence internationale, il convient d'examiner la teneur de ce discours formel et d'engager une discussion critique à cet effet. Les principaux résultats de notre analyse montrent que dans les propositions internationales liées à l'ERE, l'éducation est généralement considérée comme un instrument au service de la protection de l'environnement, qui à son tour, est généralement associé à un ensemble de problèmes de gestion des ressources, que le développement permettra de résoudre ; quant au développement lui-même, il est mal défini ou encore il est principalement associé à une croissance économique 
soutenue. Outre une discussion sur les principaux aspects de ce discours, cet article met en lumière certains éléments qui sont généralement négligés dans les propositions internationales : il s'agit de points de vue complémentaires ou divergents. L'étude que nous avons menée s'avère particulièrement pertinente au regard des réformes éducatives qui s'opèrent actuellement dans de très nombreux pays.

The great impetus provided by the UNESCO-UNEP meetings and documents for spreading, legitimising and institutionalising environmental education (EE) is incalculable. Their proposals have acted and still act as real beacons for conceiving and implementing educational reforms and programs throughout the world. While wholly acknowledging the critical importance of this kind of international support, and precisely because of its importance, it is necessary to analyse the content of the associated ruling discourse. The main findings of our analytical and critical research on international proposals related to $\mathrm{EE}$ are that education is generally seen as an instrument for the environment, itself being generally subsumed to problems of resource management to be solved through development, and development itself being ill-defined or mainly associated with sustained economic growth. Aside from identifying and discussing these main assertions, focusing on their underlying epistemological and ethical foundations, this paper also addresses elements that are generally neglected in the international proposals, as complementary or divergent viewpoints. Such an analysis is particularly relevant considering the educational reforms that are now underway in many countries.

\section{INDEX}

Mots-clés : environnement, développement, éducation relative à l'environnement, réformes éducatives, institutionnalisation, développement durable, curriculum, instrumentalisation Keywords : environnement, development, environmental education, education reforms, institutionalization, sustainable development, curriculum, instrumentalizing

\section{AUTEURS}

\section{LUCIE SAUVÉ}

Professeure au département des sciences de l'éducation de l'Université du Québec à Montréal, Lucie Sauvé est, au sein de cette institution, titulaire de la Chaire de recherche du Canada en éducation relative à l'environnement, membre de l'Institut des sciences de l'environnement et directrice du Programme d'études supérieures en éducation relative à l'environnement (ERE). Elle dirige des projets de recherche principalement axés sur l'institutionnalisation de l'ERE en milieu scolaire, sur la formation des enseignants et autres éducateurs, et sur l'intervention en milieu communautaire, tant au Québec qu'en contexte de coopération internationale.

\section{TOM BERRYMAN}

Titulaire d'un baccalauréat en biologie et d'une maîtrise en éducation, Tom Berryman a œuvré pendant de nombreuses années dans un centre d'éducation relative à l'environnement au mont Royal, à Montréal. Son mémoire de maîtrise complété en 2002 porte spécifiquement sur l'écoontogenèse. Il poursuit ses recherches au doctorat en éducation à l'UQAM où ses travaux portent sur l'éducation relative à l'environnement dans la formation initiale à l'enseignement primaire. 


\section{RENÉE BRUNELLE}

Intégrant une formation multidisciplinaire en sciences de l'environnement et en coopération internationale, Renée Brunelle mène des activités professionnelles dans le domaine de l'éducation relative à l'environnement (ERE) et de la gestion environnementale de projets à l'international. Dans le cadre de la Chaire de recherche du Canada en éducation relative à l'environnement, elle est associée à divers projets de recherche notamment sur l'institutionnalisation de l'ERE. 